\title{
DEVELOPMENT OF SDDS-COMPLIANT GENESIS AND ITS APPLICATION*
}

\author{
Yong-Chul Chae ${ }^{\dagger}$ and Robert Soliday \\ Advanced Photon Source, Argonne National Laboratory, Argonne, IL
}

\begin{abstract}
The program GENESIS has been widely used for the simulation of free-electron lasers (FELs). We extended the capabilities of the program by making the code compliant with the Self-Describing Data Set file protocol, whose toolkit has been developed and used extensively at Argonne. We report the modifications made to GENESIS and demonstrate the benefits by presenting the results obtained by its application to two major FEL projects: Argonne's Low-Energy Undulator Test Line and the Linac Coherent Light Source at SLAC.
\end{abstract}

\section{INTRODUCTION}

The program GENESIS [1] is a 3-D code simulating laser-beam interaction in the free-electron laser (FEL). It can operate in two modes: steady-state or time-dependent mode. In time-dependent mode a photon slice slips over an electron beam slice per undulator period. In this mode we can simulate self-amplified spontaneous-emission (SASE) FEL. A SASE simulation typically requires a few hundred slices, which generates large amount of data to be processed. A post-processing program based on the commercial software [2] is available from the program's author.

However, we often need to process the simulation results in a way unforeseen at the time of code development. This motivates us to convert the program compliant with Self-Describing Data Set (SDDS) file protocol [3]. Use of SDDS files has numerous advantages. One of them is the SDDS Toolkit, a software suite that works with SDDS files. It provides a complete set of postprocessing tools, including graphical display. Another advantage is that we can interface the different programs by utilizing the same data format. This enables us to perform integrated simulation that includes effects from the electron gun to the undulator based on the "Start-toEnd Simulation" (S2E) concept [4].

In the following sections we present simulation results that show new and enhanced features of SDDScompliant GENESIS.

\section{OUTPUT IN SDDS FORMAT}

The GENESIS code generates the history output along the undulator for each slice. In the SDDS-compliant version, the output consists of $m$ pages of $n$ rows; $m$ is the number of slices and $n$ is the number of locations in the

\footnotetext{
*Work supported by U.S. Department of Energy, Office of Basic Energy Sciences, under Contract No. W-31-109-ENG-38.

†chae@aps.anl.gov
}

undulator. Slice is a parameter identifying a page of data that represents a particular simulation condition. For example, if we simulate variable input wavelength, the results will be paginated according to the wavelength. We used this feature to obtain a de-tuning curve of the Linac Coherent Light Source (LCLS) FEL [5]. Table 1 shows the simulation parameters used.

Table 1: Simulation Parameters for LCLS FEL

\begin{tabular}{|c|c|}
\hline Electron Beam Energy, $\mathrm{E}_{0}$ & $14.35 \mathrm{GeV}$ \\
\hline Energy Spread, $\Delta \mathrm{E} / \mathrm{E}$ & $2 \mathrm{e}-4$ \\
\hline Peak Current, $\mathrm{I}_{\mathrm{p}}$ & $3.4 \mathrm{kA}$ \\
\hline Normalized Emittance, $\varepsilon_{\mathrm{n}}$ & $1.5 \mathrm{e}-6 \mathrm{~m}$ \\
\hline Wiggler Parameter, aw (rms) & 2.61609 \\
\hline $\begin{array}{c}\text { Matched Beta Function (Strong } \\
\text { Focusing) }\end{array}$ & $72.5 \mathrm{~m}(18 \mathrm{~m})$ \\
\hline Resonant Radiation Wavelength, $\lambda_{0}$ & $0.1492 \mathrm{~nm}$ \\
\hline Input Laser Power & $30 \mathrm{~kW}$ \\
\hline
\end{tabular}

In our simulation we scan the wavelength of input laser from $0.999 \lambda_{0}$ to $1.001 \lambda_{0}$ in 20 steps, a "do-loop" option available in GENESIS. Power and growth rate along the undulator are shown in Fig. 1, where each curve corresponds to one of the input wavelengths. We used sddsplot, an SDDS Toolkit program, for displaying the data. The command for the log-plot of Power vs. $Z$ is:

sddsplot output.sdds -column=Z,Power - split=pages mode $=y=\log , y=$ special,

where the input file is output.sdds, an SDDS-compliant GENESIS output.

In Fig. 1, the regime between $40 \mathrm{~m}$ and $80 \mathrm{~m}$ is depicted as the exponential gain regime, where the power of most slices showed exponential growth. In this regime, the power growth can be expressed as

$$
\mathrm{P}(\mathrm{Z})=\mathrm{P}_{0} \exp \left(\mathrm{Z} / \mathrm{L}_{\mathrm{g}}\right)
$$

where $\mathrm{P}_{0}$ is the initial power, $\mathrm{Z}$ is the position along the undulator, and $\mathrm{L}_{\mathrm{g}}$ is the gain length. Gain length is an important FEL parameter that should be minimized for the best performance. We can determine the gain lengths by calculating the inverse of the average value of growth rate within the exponential gain regime, as shown in the bottom of Fig. 1. 

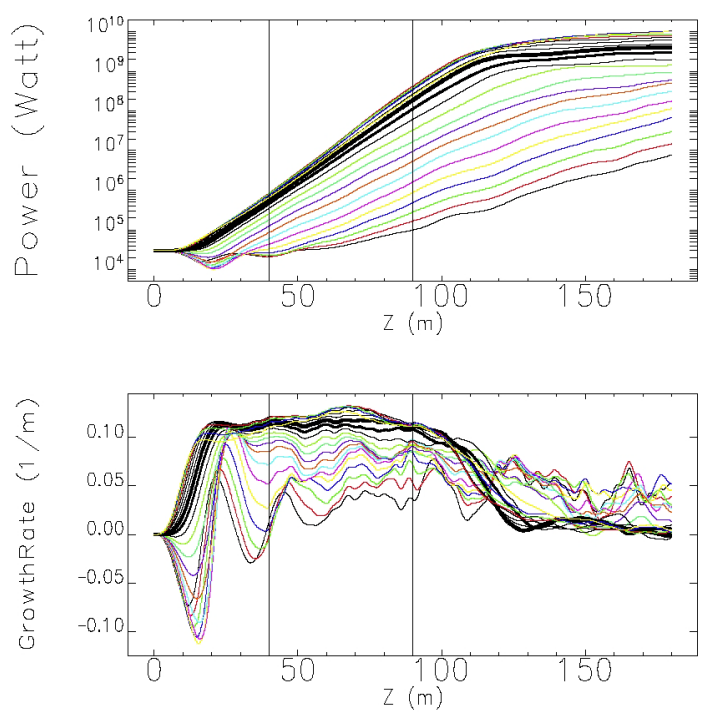

Figure 1: Power (top) and growth rate (bottom) along the undulator.

For this task we used another SDDS Toolkit program sddsprocess as shown in the command line:

\section{sddsprocess output.sdds - pipe $=$ out}

-filter $=$ column, $Z, 40,90 \mid$ sddsprocess - pipe $=$ in

GrowthRate.sdds - process $=$ column, GrowthRate, average.

This command reads GENESIS output output.sdds, filter the data in the given range between $Z=40$ and $90 \mathrm{~m}$, calculate the average of growth rate for each slice, and store the results in GrowthRate.sdds, which can be plotted by sddsplot. The resultant de-tuning curve is shown in Fig. 2, from which we find that the optimum wavelength is $1.0007 \lambda_{0}$.

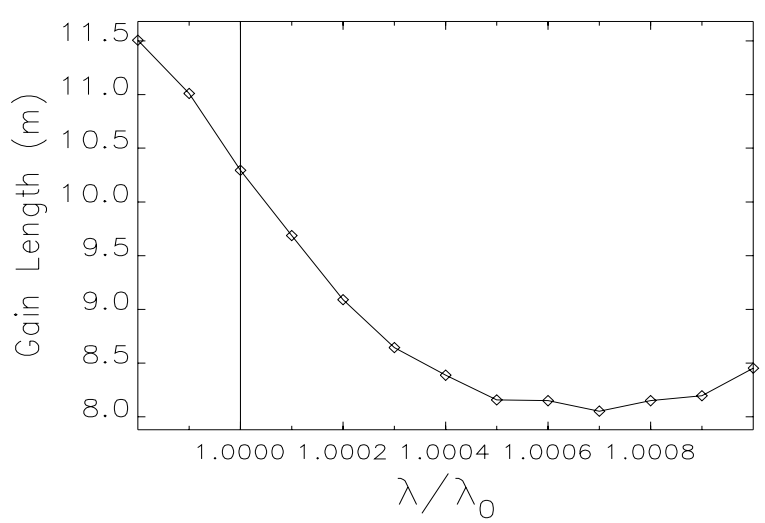

Figure 2: De-tuning curve: Gain Length vs. Wavelength.

\section{START-TO-END (S2E) SIMULATION}

Recently, an integrated simulation including effects from electron gun to undulator has been proposed and demonstrated based on the concept of "Start-to-End" simulation [4] by adopting SDDS as a common protocol between the different programs. We used the SDDScompliant GENESIS for simulating LCLS FEL in S2E.
Prior to undulators, the beam file was generated by the program elegant [6], which simulates the linac and transport line. A particle distribution from elegant simulation is shown in Fig. 3. Fig. 3a shows the longitudinal phase-space compressed by bunch compressors. Fig. $3 b$ shows the projected $x-p_{x}$ space of the whole beam distorted by wakefield in the linac. Figs. $3 \mathrm{c}$ and $3 \mathrm{~d}$ show the projected $\mathrm{x}-\mathrm{p}_{\mathrm{x}}$ space of sliced region I and II depicted in Fig. 3a. It is important to note that the sliced phase-space is in the elliptical distribution without showing severe distortion. Thus, we can approximate the slice with a few beam parameters, and, in turn, we can transfer the beam from linac to FEL, between the programs, by passing the parameters instead of the wholeparticle coordinates.
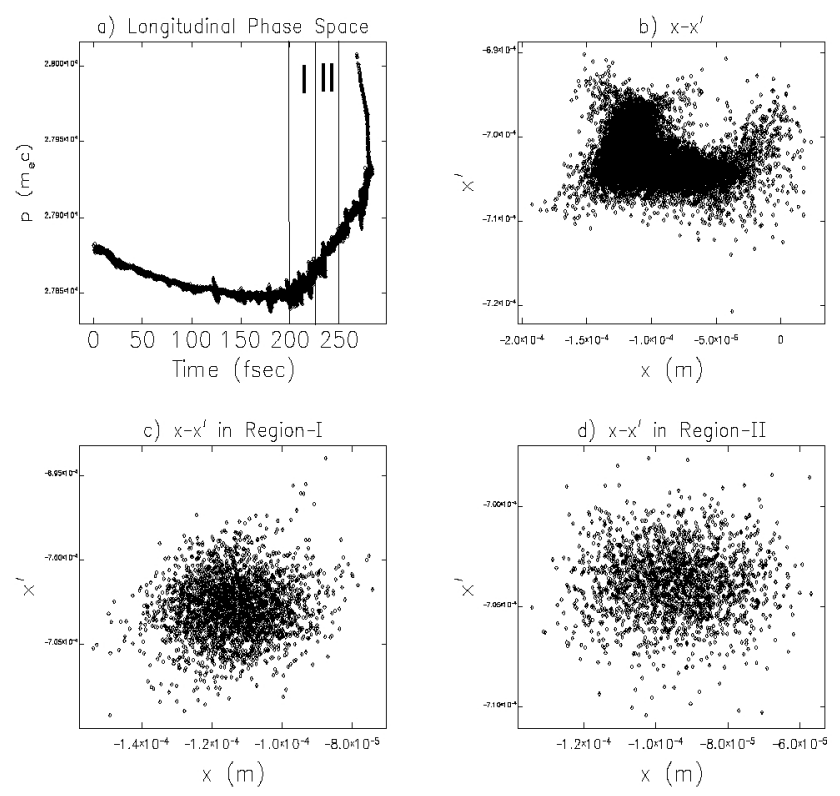

Figure 3: Particle distribution from elegant simulation of LCLS beam.
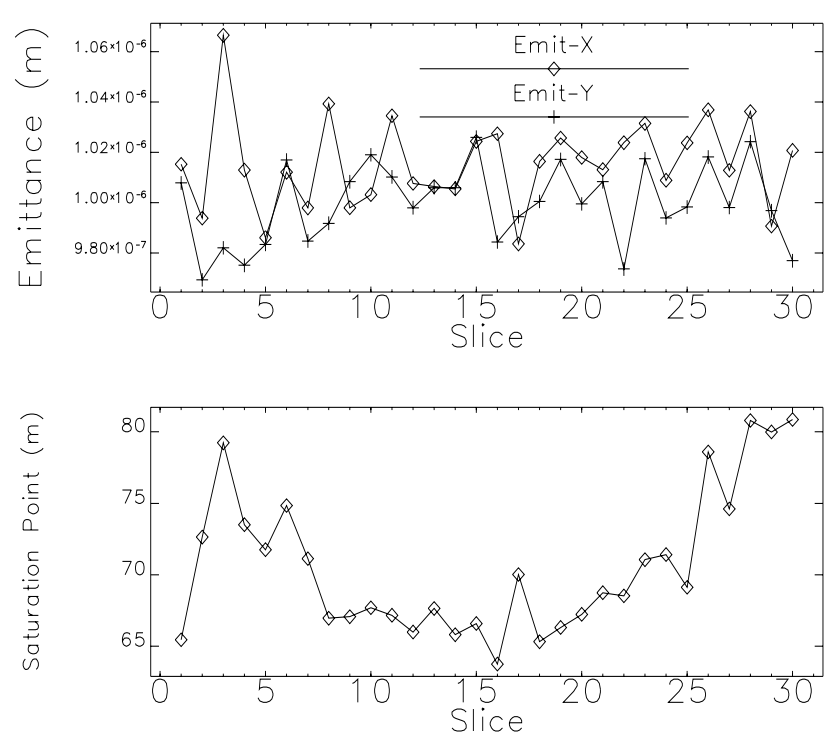

Figure 4: Emittance (top) and saturation length (bottom) of each slice along the bunch. 
An interfacing program elegant2genesis is used, which reads particle coordinates from elegant output, divides the beam in many slices, and calculates BEAMFILE parameters for each slice, such as emittance, centroid position, energy, and energy spread. GENESIS loads the particle in 6-D phase space based on the BEAMFILE parameters, then tracks through the undulators. We automated this procedure by using the SDDS-based script.

We present results simulating the LCLS FEL under strong focusing conditions. First, we show the slice emittances, a quantity in BEAMFILE (top of Fig. 4); these are the inputs to GENESIS. One performance output, saturation length vs. slice, is also shown in the bottom of Fig. 4.

\section{SIMULATION OF CHICANE}

Magnetic chicane has been used to compress an ultrarelativistic electron bunch. The bending introduces an energy dependence of the particle path length. In the linear expression this dependence can be written as

$$
\Delta \mathrm{L}=\mathrm{R}_{56}(\Delta \mathrm{E} / \mathrm{E}),
$$

where $\Delta \mathrm{L}$ is the change in path length. The length compression requires a linearly correlated energy spread. In our investigation, we used chicane to enhance the microbunching within a slice of beam in which FEL action produces a necessary correlated energy spread.

In the simulation, the particle coordinates are saved in PARTFILE after one segment of undulator. The data in PARTFILE are processed by using SDDS-based scripts for the action of chicane, which is a simple coordinate transformation via Eq. (2). These transformed particles are read by GENESIS for the new segment of FEL simulation. The repeated procedure allows us to simulate effects of multiple chicanes.

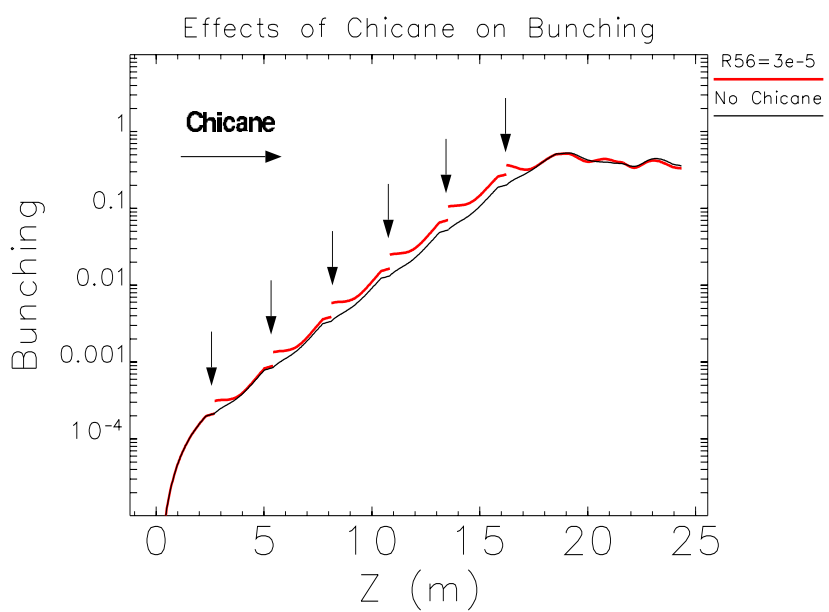

Figure 5: Effects of chicane on bunching.

We applied this to LEUTL's segmented FEL facility; the nominal LEUTL parameters are reported in [7]. The path-length parameter, $R_{56}$, was varied from zero until we found an optimal value of $\mathrm{R}_{56}=3 \mathrm{e}-5$. The effects on bunching are shown in Fig. 5, where the arrows indicate the location of chicanes. The red line is for $R_{56}=3 e-5$, whose effects are shown as the discontinuous jumps at the chicane. However, the subsequent FEL simulation shows that the beam becomes de-bunched, then follows the curve of no chicane.

\section{SASE SPECTRUM}

A recent SASE FEL experiment at LEUTL produced many interesting results, including the power spectrum along the segmented undulators. Since the power spectrum is the Fourier transform of the radiation pulse along the bunch, we rearranged the GENESIS output such that the data are along the bunch at each location of the undulator. We accomplished this by using another SDDS Toolkit program, sddsregroup, using the command:

sddsregroup ouput_z.sdds output_slice.sdds -newparameter $=Z-$ newcolumn $=$ Slice,

which reads output_z.sdds with $m$ pages of $n$ rows each, interchanges the role of column " $\mathrm{Z}$ " and the parameter "Slice", and store the rearranged data with $n$ pages of $m$ rows of each in output_slice.sdds.

Figure 6 shows the radiation pulse and power spectrum at $\mathrm{Z}=15 \mathrm{~m}$.

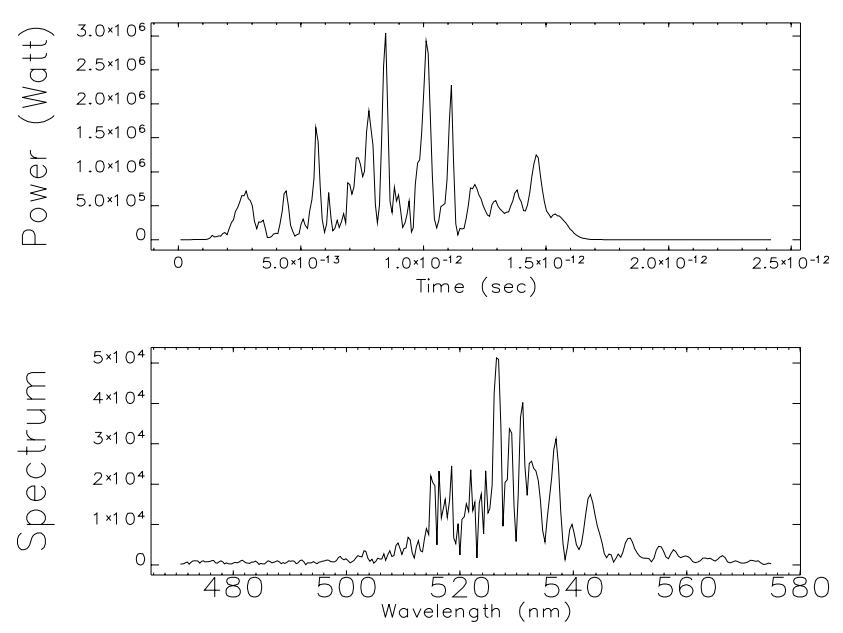

Figure 6: Radiation pulse (top) and power spectrum (bottom).

\section{REFERENCES}

[1] S. Reiche, Nucl. Instr. Meth. A 429, p. 243 (1999).

[2] IDL User's Guide, Interactive Data Language, Research Systems, Inc.

[3] M. Borland, Proc. 1995 Particle Accelerator Conference, p. 2184 (1996).

[4] M. Borland et al., "Start-to-End Jitter Simulations of the Linac Coherent Light Source," these proceedings (2001).

[5] LCLS Design Study Report, SLAC-R-521 (1998).

[6] M. Borland, ANL Report, APS/LS-287 (2000).

[7] S. Milton et al., Phys. Rev. Lett. 85, p. 988 (2000). 